\title{
Lactate- $\mathrm{H}^{+}$Transport Is a Significant Component of the In Vivo Corneal Endothelial Pump
}

\author{
Tracy T. Nguyen and Joseph A. Bonanno*
}

Purpose. To confirm the expression of monocarboxylate transporters (MCT) 1, 2, and 4 in rabbit $\mathrm{CE}$ and to test the hypothesis that cellular buffering contributed by $\mathrm{HCO}_{3}{ }^{-}$, NBCe1, and carbonic anhydrase (CA) activity facilitates lactate $-\mathrm{H}^{+}$efflux thereby controlling corneal hydration in vivo.

Methods. MCT1-4 expression of rabbit endothelium was examined by Western blotting and immunofluorescence staining. Lactate-induced acidification (LIA) was measured in perfused $\mathrm{CE}$ in the presence and absence of $\mathrm{HCO}_{3}{ }^{-}$and acetazolamide (ACTZ) using tissue treated with siRNA specific to MCT1, 2, and 4. Corneal thickness and lactate concentration were measured in New Zealand White rabbits treated with the topical CA inhibitor Azopt, and from eyes that were injected intracamerally with ouabain, disodium $4,4^{\prime}$-diisothiocyanatostilbene-2,2'-disulfonate (DIDS), and shRNA specific to the $1 \mathrm{Na}^{+}: 2 \mathrm{HCO}_{3}{ }^{-}$cotransporter NBCe1.

Results. MCT1 and MCT4 are localized to the lateral membrane, while MCT2 is apical. Cell pH measurements showed LIA in response to $40 \mathrm{mM}$ lactate in bicarbonate free (BF) Ringer's that was inhibited by niflumic acid and by MCT siRNA knockdown, and significantly reduced in the presence of $\mathrm{HCO}_{3}{ }^{-}$. Lactate-dependent proton flux in vitro was not significantly greater in the presence of $\mathrm{HCO}_{3}{ }^{-}$or reduced by ACTZ. However, when active transport, NBCe1, or CA activity was disrupted in vivo, corneal edema ensued and was associated with significant corneal lactate accumulation.

Conclusions. MCT1, 2, and 4 are expressed in rabbit CE on both the apical and basolateral surfaces and function to transport lactate- $\mathrm{H}^{+}$. Lactate- $\mathrm{H}^{+}$flux is facilitated by active transport, $\mathrm{HCO}_{3}{ }^{-}$transport and $\mathrm{CA}$ activity, disruption of which causes corneal edema in vivo and indicates that facilitation of lactate efflux is a component of the endothelial pump. (Invest Ophthalmol Vis Sci. 2012;53:2020-2029) DOI:10.1167/iovs.12-9475

$T^{1 / \epsilon}$ he corneal endothelium plays an essential role in maintaining corneal hydration and transparency. Fluid is passively imbibed into the cornea driven by the stromal

From the Indiana University School of Optometry, Bloomington, Indiana.

Supported by National Institutes of Health Grants RO1EY08834 (JAB), P30EY019008 (SB), K12EY015504 (PSS), and KO8EY019537 (TTN), and an American Optometric Foundation Ezell Fellowship (TTN).

Submitted for publication January 11, 2012; revised February 9, 2012; accepted February 16, 2012.

Disclosure: T.T. Nguyen, None; J.A. Bonanno, None

*Corresponding author: Joseph A. Bonanno, Indiana University School of Optometry, 800 East Atwater Avenue, Bloomington, IN 47405; jbonanno@indiana.edu. swelling pressure that is generated by the negatively charged stromal glycosaminoglycans. This passive fluid influx or "leak" is offset by an outward active "pump," located within the corneal endothelium, thereby maintaining corneal hydration and transparency. A breakdown in the pump function (e.g., corneal endothelial dystrophies, anterior uveitis, or trauma) will lead to corneal edema, increased light scatter, and loss of visual function.

The endothelial pump function is the composite of plasma membrane primary and secondary active transport phenomena. The pump is blocked by the $\mathrm{Na}^{+} / \mathrm{K}^{+}$-ATPase inhibitor ouabain $^{1-3}$ and is slowed in the absence of bicarbonate $e^{4-7}$ and in the presence of carbonic anhydrase inhibitors, ${ }^{4-6,8,9}$ suggesting the presence of a Na-dependent $\mathrm{HCO}_{3}{ }^{-}$linked secretory mechanism. Studies have shown the presence and activity of basolateral (stromal side) $\mathrm{Na}^{+} / \mathrm{K}^{+}$-ATPase, ${ }^{10}$ $1 \mathrm{Na}^{+}: 2 \mathrm{HCO}_{3}{ }^{-}$cotransport, ${ }^{11-14} 1 \mathrm{Na}^{+}: 1 \mathrm{~K}^{+}: 2 \mathrm{Cl}^{-}$cotransport, ${ }^{15}$ $\mathrm{Cl}^{-} / \mathrm{HCO}_{3}{ }^{-}$exchange, ${ }^{16-18}$ and $\mathrm{Na}^{+} / \mathrm{H}^{+}$exchange. ${ }^{12,16}$ However on the apical (aqueous humor facing) membrane there is no evidence of $\mathrm{Na}^{+}$or $\mathrm{Cl}^{-}$linked $\mathrm{HCO}_{3}{ }^{-}$transport. ${ }^{17,19,20}$ Regulated $\left(\mathrm{Ca}^{2+}\right.$ or cAMP) apical anion channels are present but do not contribute to basal pump activity. ${ }^{21,22}$ A recent review of the endothelial pump concludes that evidence for an $\mathrm{HCO}_{3}{ }^{-}$dependent secretory pump is lacking. ${ }^{23}$

Using cultured corneal endothelial cells, we found evidence supporting the notion that lactate- $\mathrm{H}^{+}$cotransport via monocarboxylate transporters (MCT) 1,2 , and 4 is facilitated by $\mathrm{HCO}_{3}{ }^{-}, \mathrm{CA}$ activity, $\mathrm{Na}^{+} / \mathrm{H}^{+}$exchange, and $1 \mathrm{Na}^{+}: 2 \mathrm{HCO}_{3}{ }^{-}$ cotransport. ${ }^{24}$ Eighty-five percent of the glucose taken up by the cornea is converted to two lactate molecules, ${ }^{25}$ which represents a net osmotic load. Moreover, lactate is a potent osmolyte in the cornea since additional production of lactate (e.g., during corneal hypoxia) will lead to corneal edema. ${ }^{26}$ This indicates that efficient removal of lactate is essential in maintaining corneal hydration and suggests that cellular buffering contributed by $\mathrm{HCO}_{3}{ }^{-}, \mathrm{HCO}_{3}{ }^{-}$transporters, and CA activity could be a significant component of the endothelial pump. Based on these findings, we wanted to test this hypothesis in an in vivo rabbit model. We expect that disrupting any component of the bicarbonate-carbon dioxide buffering system in vivo will lead to stromal lactate accumulation and consequently to corneal edema.

In this study, we examined the role of buffering capacity in the transport of lactic acid in Descemet's-endothelium explants ex vivo and in vivo. First, we confirmed that MCT isoforms are expressed in the rabbit endothelium and determined their membrane localization. We then examined the effect of $\mathrm{HCO}_{3}{ }^{-}$and CA activity on buffering capacity and lactate-induced cell acidification (LIA) in freshly dissected rabbit Descemet's-endothelium explants. Last, we tested the hypothesis that disrupting any component of the bicarbonate-carbon dioxide buffering system in vivo will lead to stromal lactate accumulation and consequently corneal edema. 


\section{Materials AND Methods}

\section{Immunoblotting}

New Zealand White rabbit eyes were obtained from Pel-Freez Biologicals (Rogers, AR), shipped overnight on ice, and used within 24 to 36 hours following death. Corneal scleral skirts were excised from the eyes, and the endothelium was peeled using a fine-tip forceps under a dissecting microscope. The endothelium was disrupted with RIPA lysis buffer solution $(50 \mathrm{mM}$ Tris base, $150 \mathrm{mM} \mathrm{NaCl}, 0.5 \%$ deoxycholic acid-sodium salt, $2 \%$ SDS and $1 \% \mathrm{NP} 40, \mathrm{pH} 7.5$, protease inhibitor cocktail) and sonicated (Branson 250, Branson Ultrasonics, Danbury, CT) on ice. This was followed by centrifugation at $14,000 \mathrm{~g}$ for 15 minutes to pellet cell debris. The supernatant was collected, and an aliquot was taken for protein concentration measurement using the Pierce BCA Protein Assay (Thermo Fisher Scientific, Waltham, MA). Laemmli sample buffer $(1 \times)$ was added to $15 \mu \mathrm{g}$ protein samples, and the mixture was heated in a $95^{\circ} \mathrm{C}$ heating block for 5 minutes to denature the protein. Samples were resolved on 10\% SDS-PAGE and transferred to polyvinylidene difluoride membranes (Bio-Rad, Hercules, CA). Blots were then probed with MCT1, 2, 3, or 4 polyclonal antibodies (sc-50325; sc-50323; sc-14930; and sc-50329; respectively, 1:1000 dilution; Santa Cruz Biotechnology, Santa Cruz, CA) for 1 hour at room temperature. Secondary horseradish peroxidase conjugated antibody incubation was done for 1 hour at room temperature (1:5000 dilution). Bound antibody was detected using Supersignal West Pico Chemiluminescent substrate (Pierce, Thermo Fisher Scientific).

\section{Immunofluorescence}

Immunofluorescence staining was performed on freshly peeled corneal endothelium that was placed with the apical surface up on a glass microscope slide and fixed with PLP fixation buffer solution (2\% paraformaldehyde, $75 \mathrm{mM}$ lysine, $10 \mathrm{mM}$ sodium periodate, and $45 \mathrm{mM}$ sodium phosphate, $\mathrm{pH} 7.4$ ) at room temperature for 20 minutes. Rabbit anti-MCT1, MCT2, and MCT4 polyclonal antibodies were diluted 1:50 in equal mixture of PBS-goat serum and applied to slides at room temperature for 1 hour. Goat anti-MCT3 polyclonal antibody was diluted 1:100. Coverslips were washed three times for 15 minutes in PBS containing $0.01 \%$ saponin. Then, secondary antibody, Alexa 488 anti-rabbit for MCT1, MCT2, and MCT4 and Alexa 488 anti-goat for MCT3 were applied for 1 hour at room temperature (1:1000 dilution). Slides were washed, nuclei counterstained with DAPI, and mounted with Prolong antifade medium (Molecular Probes, Invitrogen Life Technologies, Carlsbad, CA). Fluorescence was observed with an epifluorescence microscope equipped with a cooled charged couple device (CCD) camera.

\section{Measurement of Intracellular pH}

A corneal scleral skirt was dissected and used to trephine a 10-mm corneal button. The button was loaded with the pH-sensitive fluorescent dye $2^{\prime} 7^{\prime}$-bis(2-carboxyethel)-5(6)-carboxyfluorescein (BCECF) by incubation in $\mathrm{HCO}_{3}{ }^{-}$free Ringer's solution containing 1 to $5 \mu \mathrm{M}$ BCECF-acetoxymethyl (AM) at room temperature for 30 minutes. BCECF-AM was removed and replaced with $\mathrm{HCO}_{3}{ }^{-}$free Ringer's solution for at least 30 minutes before use. The corneal endothelium was peeled from the button and placed onto a 13-mm diameter, $45-\mu \mathrm{m}$ thick, $0.2-\mu \mathrm{m}$-pore diameter, rigid Anodisc filter (Whatman, Kent, UK) and mounted in a double-sided perfusion chamber. ${ }^{17}$ Fluorescence was excited alternately at $495 \pm 10 \mathrm{~nm}$ and $440 \pm 10 \mathrm{~nm}$. Fluorescence emission $(520-550 \mathrm{~nm})$ ratios $\left(\mathrm{F}_{495} / \mathrm{F}_{440}\right)$ obtained at $1 \mathrm{~Hz}$ were calibrated against $\mathrm{pH}_{\mathrm{i}}$ by the high- $\mathrm{K}^{+}$-nigericin technique. ${ }^{12,17}$ The assembled perfusion chamber was placed on a water-jacketed $\left(37^{\circ} \mathrm{C}\right)$ brass collar held on the stage of an inverted microscope (Diaphot 200; Nikon, Tokyo, Japan) and viewed with a long working distance $(2 \mathrm{~mm})$ water-immersion objective $(40 \times$; Nikon). Apical and basolateral compartments were connected by tubing (Phar-Med; Saint-Gobain Performance Plastics, Paris, France) to hanging syringes that contained Ringer's solution in a warming box $\left(37^{\circ} \mathrm{C}\right.$ ). The flow of the perfusate (approximately $0.5 \mathrm{~mL} / \mathrm{min}$ ) was achieved by gravity. Two independent eight-way valves were used to select the desired perfusate for the apical and basolateral chambers. We used Felix32 (Photon Technology International, Birmingham, NJ) software to analyze the $\mathrm{pH}_{\mathrm{i}}$ change. The initial rate of $\mathrm{pH}_{\mathrm{i}}$ change taken over the first 20 seconds was used to determine $\mathrm{dpH}_{\mathrm{i}} / \mathrm{dt}$. The rate of acidification is expressed as $\mathrm{pH}_{\mathrm{i}} / \mathrm{s}$, and the degree of acidification was determined by the amount of change in $\mathrm{pH}_{\mathrm{i}}$ and is expressed as $\Delta \mathrm{pH}_{\mathrm{i}}$.

\section{Ringer's Solutions and Chemicals}

The composition of the standard bicarbonate-free Ringer's solution used throughout this study was (in millimoles) $150 \mathrm{Na}^{+}, 4 \mathrm{~K}^{+}, 0.6$ $\mathrm{Mg}^{2+}, 1.4 \mathrm{Ca}^{2+}, 118 \mathrm{Cl}^{-}, 1 \mathrm{HPO}_{4}^{2-}, 10 \mathrm{HEPES}, 28.5$ gluconate ${ }^{-}$, and 5 glucose. Bicarbonate-rich Ringer's solution was prepared by equimolar substitution of sodium gluconate with $\mathrm{NaHCO}_{3}{ }^{-}(28.5 \mathrm{mM})$. Ringer's solutions were equilibrated with air (BF) or $5 \% \mathrm{CO}_{2}(\mathrm{BR})$, and $\mathrm{pH}$ was adjusted to 7.5 with $\mathrm{NaOH}$ at $37^{\circ} \mathrm{C}$. Lactate solutions were prepared on the morning of the experiment by equimolar substitution of $\mathrm{Na}^{+}$gluconate by the $\mathrm{Na}^{+}$-salt of $\mathrm{L}(+)$ lactate. Osmolarity of all solutions was adjusted to 295 to 300 mOsm by adding sucrose. Sodium $\mathrm{L}(+)$ lactate, niflumic acid, disodium 4,4'-diisothiocyanatostilbene-2,2'-disulfonate (DIDS), ouabain, and acetazolamide (ACTZ) were obtained from Sigma Aldrich (St. Louis, MO). BCECF-AM was purchased from Molecular Probes.

\section{MCT Knockdown Ex Vivo}

MCT1, 2, and 4 specific siRNAs were purchased from Santa Cruz Biotechnology (Catalog Nos.: MCT1, sc-108031; MCT2, sc-108032; and MCT4, sc-108033). Ex vivo cornea cultures were established by the following procedure. New Zealand White rabbit eyes were sterilized with $1 \%$ povidone iodine for 1 minute and rinsed with sterile PBS. A corneal scleral skirt was excised aseptically and washed with sterile PBS, then placed in a sterile six-well plate with adaptations that allow the tissue to sit upright and remain cupped. MCT 1, 2, or 4 siRNA was added along with lipofectamine 2000 in $100 \mu \mathrm{L}$ OPTI-MEM (Gibco, Invitrogen, Grand Island, NY) to the endothelial surface and incubated for 6 hours at $37^{\circ} \mathrm{C}, 5 \% \mathrm{CO}_{2}-95 \%$ air. The transfection medium was removed, and the tissue was placed in a sterile 12-well plate and immersed with $2 \mathrm{~mL}$ Dulbecco Modified Eagle Medium (DMEM), 10\% bovine calf serum (BCS) and $1 \%$ antibiotic/antimycotic $(100 \mathrm{U} / \mathrm{mL}$ penicillin and $0.25 \mathrm{ug} / \mathrm{mL}$ Fungizone). The medium was changed twice a day. Seventy-two hours post transfection, the endothelium was peeled and LIA was measured. A nontargeting siRNA (Dharmacon, Thermo Fisher Scientific) was used as negative control.

\section{Experimental Animals}

New Zealand White rabbits (8-10 weeks old, 4-5 lbs) were obtained from Harlan Laboratories (Indianapolis, IN) and used as the in vivo animal model. All animal procedures were performed in accordance with the Declaration of Helsinki, the Indiana University Animal Care and Use Committee, and the ARVO Statement for the Use Animals in Ophthalmic and Vision Research. For anterior chamber injections, rabbits were sedated with ketamine $\mathrm{HCl}(30 \mathrm{mg} / \mathrm{kg}$, Fort Dodge, IA) and xylazine $\mathrm{HCl}(5 \mathrm{mg} / \mathrm{kg}$, intramuscular [IM], Fort Dodge, IA). Two drops of topical anesthesia $(0.5 \%$ proparacaine $\mathrm{HCl}$; Akorn, Lake Forest, IL) were used for anterior chamber injections and central corneal thickness (CT) measurements. For euthanasia, rabbits were sedated and then euthanized by injection of $2.5 \mathrm{~mL}$ Sleepaway sodium pentobarbital euthanasia solution (Fort Dodge, IA) intracardially. 


\section{Intracameral Injection of Pharmacologic Inhibitors}

After sedating the rabbit, two drops of $0.5 \%$ proparacaine $\mathrm{HCl}$ were topically applied to the eye. With the aid of an ophthalmic surgical microscope, $25 \mu \mathrm{L}$ of $1-\mathrm{mM}$ ouabain or $25 \mu \mathrm{L}$ of $10-\mathrm{mM}$ DIDS was injected into the anterior chamber of the left eye using a 30-gauge needle on a Hamilton glass syringe (Hamilton Company, Reno, NV). The right eye was injected with $25 \mu \mathrm{L}$ sterile PBS. The needle was inserted perpendicular to the corneal surface 1 to $2 \mathrm{~mm}$ from the limbus, and then while the tip was still in the stroma, turned and pointed toward the anterior-center of the anterior chamber. The inhibitor was slowly injected into the anterior chamber, and the needle was carefully removed from the cornea.

\section{Central Corneal Thickness Measurements}

CT measurements were made by ultrasound pachymetry (DGH Technology, Exton, PA). Two drops of proparacaine were topically applied on the corneas of unsedated rabbits. Baseline corneal thickness measurements were made before injection of the pharmacologic inhibitors or viral particles. Subsequently, after injection with the pharmacologic inhibitors, CT measurements were made hourly until peak CT was obtained (between 5 and 7 hours). CT measurements were made weekly on eyes injected with viral particles.

\section{NBCe1 In Vivo Knockdown}

Rabbits were injected intracamerally with the electrogenic sodium bicarbonate cotransporter $\left(1 \mathrm{Na}^{+}: 2 \mathrm{HCO}_{3}{ }^{-}\right)$shRNA (NBCe1; SLC $\left.4 a 4\right)$ as part of a previous study. ${ }^{27}$ The eyes transfected with NBCe1 shRNA that had greater than $25 \%$ knockdown after 2 to 4 weeks were used to examine the association between corneal edema and lactate concentration. Briefly, lentiviruses were used to deliver the NBCe1 and scrambled shRNA into the corneal endothelium. The details of the shRNA sequences, technique for lentivirus production, and anterior chamber injection have been previously described. ${ }^{27}$ Baseline CT measurements were made prior to the intracameral injection and weekly post injection. Following euthanasia, the eyes were enucleated, and the corneal endothelium was peeled and subjected to Western blotting to determine efficiency of knockdown. The remaining cornea was used to analyze lactate concentration following the procedure described by Klyce ${ }^{26}$ with some minor modifications. Briefly, a 10-mm central corneal button was trephined from the tissue and immediately placed in a mortar and submerged with liquid nitrogen for 1 minute to stop further metabolism. With a steady stream of liquid nitrogen pouring over the tissue, it was pulverized using a pestle. The sample was then transferred to an Eppendorf tube (Eppendorf, Hauppauge, $\mathrm{NY}$ ) filled with $1 \mathrm{~mL}$ PBS, centrifuged at $1700 \mathrm{~g}$ for 15 minutes, and the supernatant collected for lactate analysis. The pellet was used to determine protein concentration. One-hundred microliters RIPA lysis buffer was added to the pellet and sonicated on ice. This was followed by centrifugation at $14,000 \mathrm{~g}$ for 15 minutes to pellet cell debris. The supernatant was collected, and an aliquot was taken for protein concentration measurement using the Pierce BCA Protein Assay (Thermo Scientific).

\section{Statistical Analysis}

Data analysis is presented as mean values \pm standard error. Paired $t$-test was used for statistical analysis and $P<0.05$ was considered significant.

\section{Results}

As previously reported for the bovine corneal endothelium, ${ }^{24}$ Western blotting confirmed that MCT1, MCT2, and MCT4 are expressed in the rabbit corneal endothelium (Fig. 1A). Similar to bovine, MCT3 was not expressed. Immunofluorescent staining localized MCT1 and MCT4 to the basolateral and MCT2 to the apical surface (Fig. 1B). To test if MCTs are functional in the rabbit corneal endothelium, we measured the amount of acidification during 1-minute exposure to $40 \mathrm{mM}$ lactate on the BL surface in the absence and presence of 100 $\mu \mathrm{M}$ niflumic acid, a nonspecific MCT inhibitor (Fig. 1C). Niflumic acid on the BL surface in BF Ringer's solution reduced the rate of LIA by $57 \%(n=6, P=0.0008)$ and amount of LIA by $41 \%$ ( $n=6, P=0.0007$ ) (Fig. 1D).

To further confirm that MCTs are directly involved in transporting lactate- $\mathrm{H}^{+}$, LIA was measured 72 hours after siRNA knockdown of MCT1, MCT2, and MCT4 collectively within ex vivo cultured corneas. Maximum knockdown of MCT1 was achieved with $200 \mathrm{nM}$ siRNA (62\% knockdown) and MCT4 and MCT2 with $100 \mathrm{nM}$ siRNA (83\% and 16\% knockdown, respectively) (Figs. 2A-C). Intracellular $\mathrm{pH}$ was determined in $\mathrm{CE}$ that was treated with a combination of MCT1, MCT2, and MCT4 siRNAs and on CE treated with scrambled siRNA. Twenty millimolar lactate pulses in BF Ringer's solution were simultaneously given on both the apical and basolateral surfaces for 25 seconds. Compared with cells treated with scrambled siRNA, the rate of acidification was slowed by $55 \%$ in cells treated with MCT siRNAs $(n=8, P=$ $0.002)$, and the amount of acidification was reduced by $78 \%$ ( $n$ $=8, P=0.0002$ ) (Fig. 2D), indicating that the MCTs are responsible for LIA. When lactate was perfused only on the $\mathrm{BL}$ surface in cells treated with MCT siRNAs, there was no LIA (data not shown), consistent with the much greater knockdown of the basolateral MCT1 and 4 .

Previously, we demonstrated that bicarbonate and carbonic anhydrase enhanced lactate- $\mathrm{H}^{+}$cotransport in cultured bovine corneal endothelial cells (CEC). ${ }^{24}$ We attributed the facilitated transport to increased intracellular buffering capacity. To determine if this mechanism was also significant in rabbit endothelium, we first examined the effect of bicarbonate on lactate-induced acidification. In the absence of bicarbonate, there was significant acidification when $20 \mathrm{mM}$ lactate was perfused for 1 minute on either the apical or basolateral surface (Fig. 3A). However, in the presence of bicarbonate, the amount of acidification was significantly less on the basolateral surface and unmeasurable on the apical surface (Fig. 3A). Next, we decreased intracellular buffering by incubating the tissue in $100 \mu \mathrm{M}$ acetazolamide, a carbonic anhydrase inhibitor. Acetazolamide did not change the rate of LIA in tissues perfused without bicarbonate (0.00167-0.00163 pH units/sec, $n=5, P=0.57$ ) (Fig. 3B). However, in the presence of bicarbonate, acetazolamide produced a small, but significant increase in the rate of LIA on the BL surface (from 0.0006 to $0.0008 \mathrm{pH}$ units/sec, $n=8, P=0.03$ ) (Fig. 3B). To determine if lactate-dependent proton flux $\left(\mathrm{J}_{\mathrm{H}}{ }^{+}[\mathrm{mM} / \mathrm{sec}]=\beta_{\mathrm{T}}{ }^{*} \mathrm{dpH}_{\mathrm{i}} / \mathrm{dt}\right)$ was affected by $\mathrm{HCO}_{3}{ }^{-}$or ACTZ, total buffering capacity $\left(\beta_{\mathrm{T}}=\right.$ $\left.\beta_{\mathrm{i}}+\beta_{\mathrm{HCO} 3}\right)$ was determined under each condition as previously described. ${ }^{12,24}$ The intrinsic buffering capacity $\left(\beta_{\mathrm{i}}\right.$ $=20 \mathrm{mM} / \mathrm{pH}$ ) of the rabbit endothelium was determined using $\mathrm{dpH}_{\mathrm{i}} / \mathrm{dt}$ of the first 20 seconds following exposure to $\mathrm{NH}_{4} \mathrm{Cl}^{12}$ We found that $\beta_{\mathrm{HCO} 3}\left(=2.303 *\left[\mathrm{HCO}_{3}{ }^{-}\right]_{\mathrm{i}}\right)^{28}$ was reduced by $43 \%$ in the presence of ACTZ. This relative reduction in buffering was experimentally determined by measuring the change in $\mathrm{pH}_{\mathrm{i}}$ during a pulse of $2 \mathrm{mM} \mathrm{NH} \mathrm{NH}_{4} \mathrm{Cl}$ the absence and presence of $100 \mu \mathrm{M}$ ACTZ in BR Ringer's solution (data not shown). Although the rate of LIA was significantly smaller in BR Ringer's solution, with the increased buffering supplied by bicarbonate, lactate-dependent proton flux was 13\% greater; however, this was not significant $(P>0.05$; Table 1$)$. Table 1 also shows that ACTZ had no effect on lactate-dependent proton flux in the absence of $\mathrm{HCO}_{3}{ }^{-}$as expected; however, in the presence of $\mathrm{HCO}_{3}{ }^{-}$proton flux decreased by $6 \%$, but again 
A

B
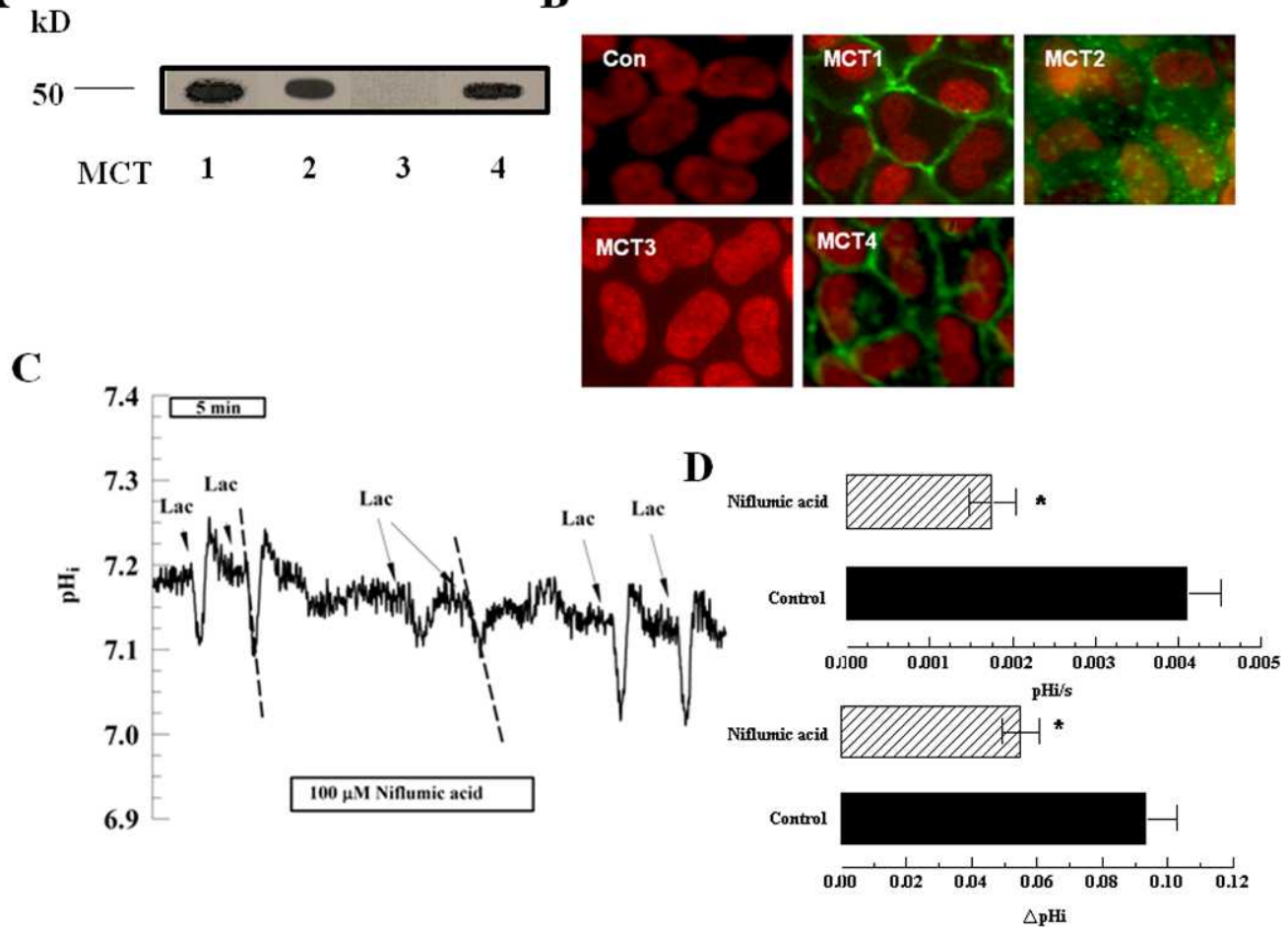

Figure 1. Expression, localization and function of MCTs in rabbit corneal endothelium. (A) Whole cell lysates from rabbit CE were resolved using $10 \%$ SDS-PAGE gel. Fifteen micrograms of total protein was loaded per lane. MCT1, 2, and 4, but not MCT3, were immunodetected at approximately $50 \mathrm{kD}$. (B) Immunofluorescent staining was performed on freshly dissected rabbit endothelium-Descemet's preparations. Panel B shows representative immunofluorescent images for MCT1-4. Control samples were not subjected to the respective MCT primary antibodies. Overlay images: red, nuclei; green, MCT. MCT1 and MCT4 show lateral staining, while MCT2 shows apical staining. (C) Basolateral lactate (40 mM) induces cell acidification (arrows) that is inhibited by $100 \mu \mathrm{M}$ niflumic acid. The dashed lines represent the estimate of the initial dpH/dt. (D) Mean acidification rates and the mean change in $\mathrm{pH}_{\mathrm{i}}(n=6) \pm \mathrm{SEM}$ due to lactate. Rates were determined over the first 20 seconds. The mean rate was reduced by $57 \%$, and LIA was decreased by $41 \%$ in the presence of niflumic acid. * Significantly lower than control $(P<0.05)$.

not significant $(P>0.05)$. So, unlike the cultured bovine corneal endothelium, ${ }^{24}$ we could not demonstrate significant $\mathrm{HCO}_{3}{ }^{-} / \mathrm{CA}$-facilitated lactate-dependent $\mathrm{H}^{+}$fluxes in vitro in the rabbit endothelium. A major difference is that the rate of LIA in the rabbit endothelium is more than 10-fold smaller (e.g., $\mathrm{dpH} / \mathrm{sec}=0.011$ in bovine cells and 0.0008 in rabbit in $\mathrm{BR}+$ ACTZ), which significantly limits the ability to resolve differences. Therefore, we next explored disruption of $\mathrm{HCO}_{3}{ }^{-}$ buffering using an in vivo approach.

If the components of the cellular buffering system facilitate the removal of lactate in vivo, then disrupting the function of these components will lead to slower clearance of lactate from the cornea and accumulation of stromal lactate will produce corneal edema. Hence, we disrupted components of the cellular buffering system using pharmacologic inhibitors and/ or shRNA, and measured the change in corneal thickness and corneal lactate concentration. First, we predict that inhibiting the sodium-potassium ATPase, which produces the cell membrane $\mathrm{Na}^{+}$gradient that drives secondary active transporters such as $\mathrm{Na}^{+}: 2 \mathrm{HCO}_{3}{ }^{-}$cotransport and $\mathrm{Na}^{+} / \mathrm{H}^{+}$ exchange will affect lactate removal. Ouabain $(25 \mu \mathrm{L}, 1 \mathrm{mM})$ was injected intracamerally in one eye of seven rabbits, and the other eye received $25 \mu \mathrm{L}$ PBS. Corneal swelling was noted in all ouabain-injected eyes peaking at approximately 5 hours. No change in corneal thickness was noted in the control eyes. The mean corneal swelling response was $23 \% \pm 3 \%$. Eyes injected with ouabain showed a significant 1.82 -fold increase in corneal [lactate] (Fig. $4 \mathrm{~A}, P=0.02$ ). There was a moderate correlation $(r=+0.57)$ between the extent of corneal swelling and [lactate] in eyes treated with ouabain, although this correlation was not significant, $P>0.05$ (Fig. 4A). Previously, we had shown that two drops of the topical CA inhibitor Azopt (1\%, brinzolamide ophthalmic suspension [Alcon, Fort Worth, TX]) caused corneal swelling in the rabbit. ${ }^{27}$ Azopt produced $6 \% \pm 0.006 \%$ corneal swelling at 3 hours and a significant 1.43 -fold increase in corneal lactate concentration ( $n=7, P=0.01$ ) relative to control eyes (Fig. $4 \mathrm{~B})$. However, the correlation between the extent of corneal swelling and [lactate] in eyes treated with Azopt was weak $(r$ $=+0.22, P>0.05$; Fig. $4 \mathrm{~B})$.

Next, we examined the role of $\mathrm{HCO}_{3}{ }^{-}$transport in facilitating lactate- $\mathrm{H}^{+}$cotransport in vivo. We disrupted the basolateral $\mathrm{Na}^{+}: 2 \mathrm{HCO}_{3}{ }^{-}$cotransporter using the nonspecific anion transport inhibitor DIDS or via NBCe1 shRNA delivered intracamerally with lentiviruses. Relative to the PBS-injected eyes, DIDS-treated eyes showed $22 \% \pm 2 \%$ corneal swelling at 5 hours and a significant 1.25 -fold greater corneal [lactate] compared with the untreated eye $(n=6, P=0.03)$ (Fig. 5A). There was a moderate $(r=+0.50)$, but not statistically significant $(P>0.05)$, correlation between corneal swelling and [lactate] in eyes treated with DIDS (Fig. 5B). Of the 11 rabbits injected with NBCe1 shRNA, 8 rabbits had $>25 \%$ knockdown and were included in the analysis. Corneal [lactate] was significantly increased by two-fold in the NBCe1 knockdown eyes (Fig. 6A, $P<0.05$ ). There was a strong correlation $(r=+0.81, P<0.01)$ between corneal swelling and [lactate] (Fig. 6B). 


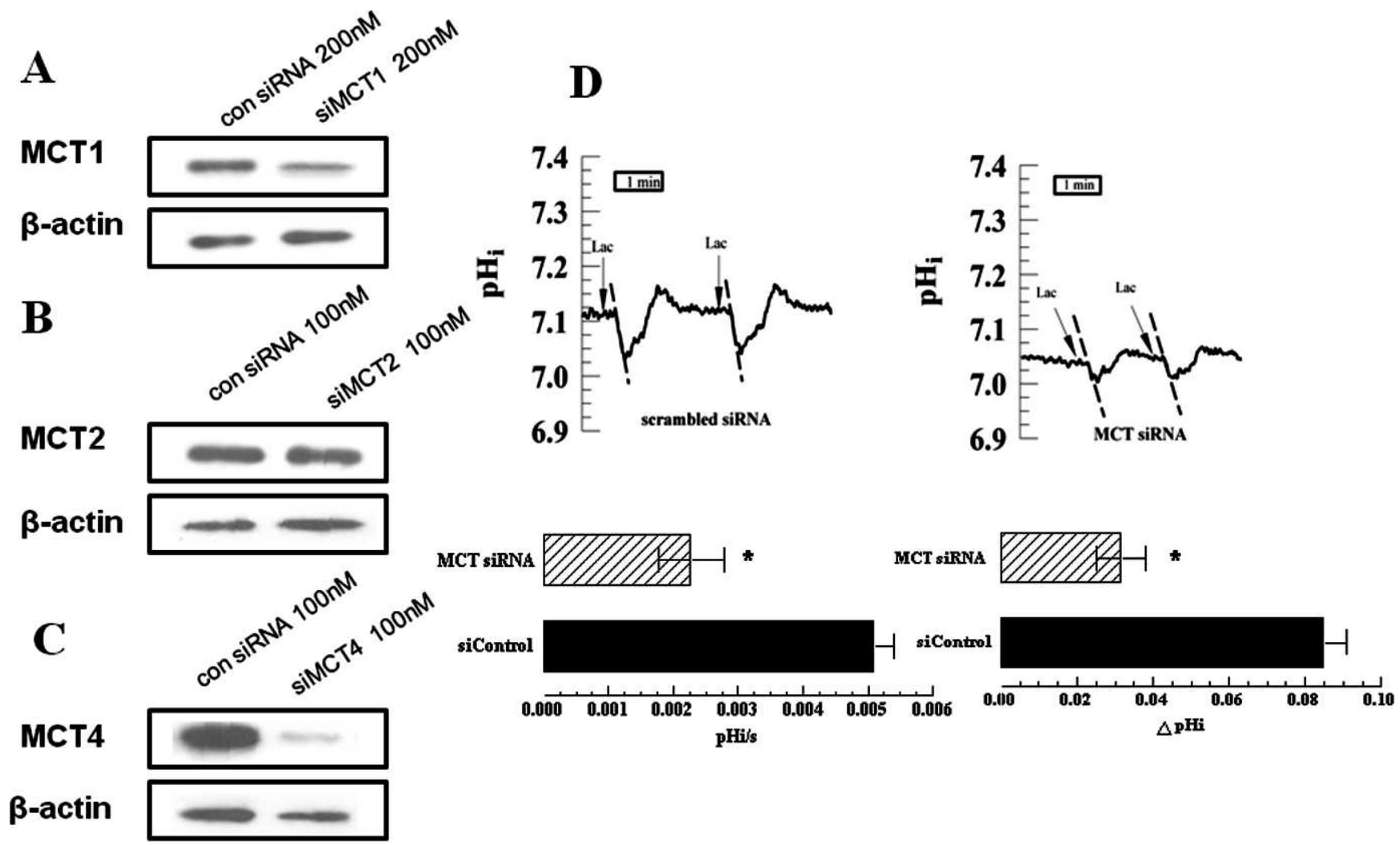

Figure 2. Knockdown of MCT1, 2, and 4 with siRNA in ex vivo corneas. Panels A-C are Western blot images that show maximum knockdown concentration for each MCT at 72 hours post transfection. Ten micrograms of protein was loaded per lane. Sixty-two percent, $16 \%$, and $83 \%$ knockdown was achieved with MCT1, MCT2, and MCT4, respectively. Panel D shows that compared with the cells treated with scrambled siRNA, the rate of LIA was $55 \%$ slower in cells treated with MCT siRNAs, and the amount of LIA was reduced by $78 \%(n=8) .{ }^{*}$ Significantly lower than control $(P<0.05)$.

\section{Discussion}

In the current study, we determined that MCT 1, 2, and 4 are expressed in the rabbit corneal endothelium on apical and basolateral membranes. The MCTs function to move lactate coupled to $\mathrm{a} \mathrm{H}^{+}$in a direction determined by the sum of the chemical gradients. The lactate concentration gradient from stroma (approximately $14 \mathrm{mM}$ ) to anterior chamber (approximately $7 \mathrm{mM})^{26}$ is 2 , and the proton gradient is variable, but in the same direction, ${ }^{29,30}$ Therefore, the MCTs will facilitate lactate efflux across the endothelium to the anterior chamber. The presence of bicarbonate and CA activity significantly buffers $\mathrm{pH}_{\mathrm{i}}$, but in contrast to cultured $\mathrm{CE},{ }^{24}$ we showed only a modest facilitation of lactate: $\mathrm{H}^{+}$cotransport in vitro. On the other hand, disruption of active transport, pHi regulation and buffering in vivo via inhibition of sodium-potassium ATPase, carbonic anhydrase, or $\mathrm{Na}^{+}: 2 \mathrm{HCO}_{3}{ }^{-}$cotransporter produced significant corneal edema and stromal lactate accumulation.

Of interest, the MCT expression pattern in rabbit endothelium was slightly different from cultured bovine CE. ${ }^{24}$ In rabbit, MCT1 and 4 were basolateral and MCT2 apical. Whereas in bovine, MCT4 was apical and MCT2 basolateral. ${ }^{24}$ MCT4 has a basolateral sorting signal in the C-terminal portion of the cytoplasmic tail. ${ }^{31}$ Nevertheless, some studies show that MCT4 can localize to the apical membranes in certain tissues. ${ }^{32,33}$ Even in the same organ (e.g., small and large intestine), the expression profile of MCT4 varies depending on the region. ${ }^{33}$ It has also been reported that MCT expression in a tissue can vary among different animal species. ${ }^{34}$ This may be related to differential expression of the needed chaperones between bovine and rabbit endothelium. ${ }^{35,36}$ Further studies are needed to understand these discrepancies.

As in our previous study, ${ }^{24}$ we show that niflumic acid, a well-described inhibitor of MCTs, ${ }^{37}$ inhibits LIA. Niflumic acid is nonspecific and has been shown to also inhibit anion channels, specifically $\mathrm{Cl}^{-}$channels leading to membrane hyperpolarization. ${ }^{38}$ However, lactate: $\mathrm{H}^{+}$cotransport is electroneutral, so a secondary effect on lactate-coupled proton influx is remote. In our experiment, we compared the acidification of cells in response to a lactate pulse in the absence and presence of niflumic acid. We showed a significant inhibition of acidification, which is consistent with niflumic acid's known inhibitory action on MCTs. AstraZeneca (Wilmington, DE) has developed compounds that show very specific inhibition of MCT1 and 4; however, these compounds are currently unavailable (A. Halestrap, written communication, 2011).

To provide direct and specific evidence for the action of MCTs in rabbit endothelium, siRNAs were used to silence MCT1, 2, and 4 in ex vivo corneas. Although we did not achieve complete knockdown of the MCTs, we were able to reduce expression of all three MCTs and show corresponding reduction in LIA when we perfused with lactate on both the basolateral and apical surfaces simultaneously (Fig. 2D). However, when lactate was perfused only on the BL surface, there was no LIA, indicating that the observed LIA in cells treated with the siRNAs was predominantly via apical lactate$\mathrm{H}^{+}$cotransport from MCT2, which was knocked down by only $16 \%$. In sum, these experiments demonstrate that MCTs are responsible for lactate-induced acidification. 

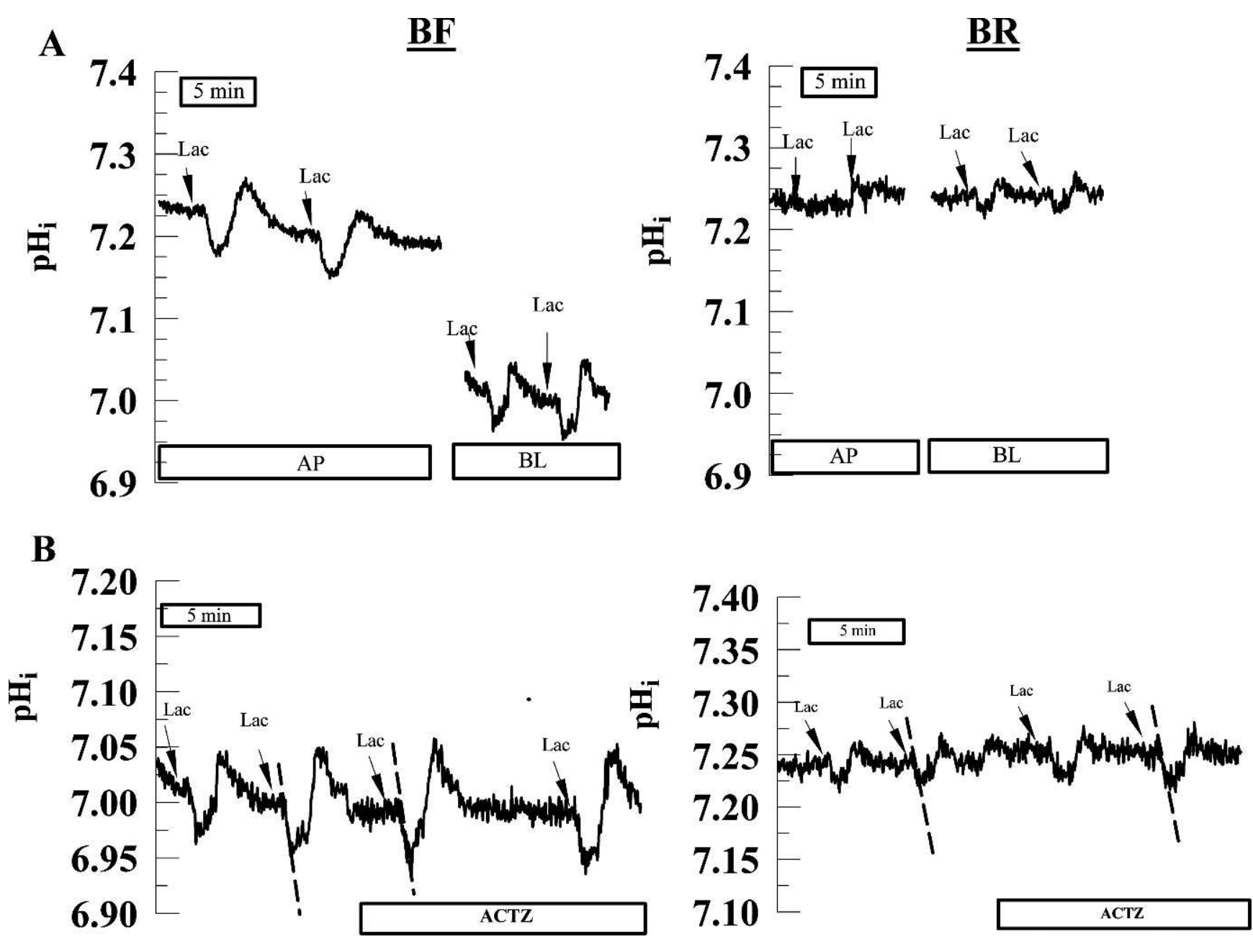

FiguRE 3. Lactate-induced acidification in BF and BR Ringer's solution and in the presence of acetazolamide (ACTZ), a CA inhibitor. These are representative traces of the change in $\mathrm{pH}_{\mathrm{i}}$ induced by $20 \mathrm{mM}$ lactate in $\mathrm{BF}$ and $\mathrm{BR}$ medium and in the absence and presence of $100 \mu \mathrm{M}$ ACTZ. Arrows indicate where lactate pulses were given. (A) There was significant decrease in $\mathrm{pH}_{\mathrm{i}}$ in when lactate was perfused for 1 minute on either the $\mathrm{BL}$ or apical surface in BF Ringer's solution. The results are from two different CE samples. There was little (BL surface) or no (Apical) LIA when the CE were perfused in BR Ringer's solution. (B) The rate of acidification was not affected by ACTZ in BF Ringer's solution; however, in BR Ringer's solution, acidification was slightly faster (from 0.0006 to $0.0008 \mathrm{pH}$ units/sec). The dashed lines are estimates of the slope.

Previously we had shown that bicarbonate buffering and carbonic anhydrase activity facilitate lactate: $\mathrm{H}^{+}$cotransport in cultured bovine corneal endothelial cells. ${ }^{24}$ Moreover, other studies have shown that MCT-dependent lactate- $\mathrm{H}^{+}$flux is facilitated by bicarbonate transporters and carbonic anhydrase (CA) activity in various cells and tissue. ${ }^{39-45}$ Here, we found that LIA in freshly dissected rabbit CE was more than 10-fold less than in bovine cells in bicarbonate-free conditions and approximately 30-fold less in bicarbonate-rich Ringer's solution. Total buffering capacity in rabbit CE is only slightly greater than cultured bovine endothelium, indicating that overall lactate: $\mathrm{H}^{+}$fluxes are much less in the fresh rabbit $\mathrm{CE}$, making it difficult to examine the effects of buffering on lactate: $\mathrm{H}^{+}$cotransport by measuring changes in $\mathrm{pH}_{\mathrm{i}}$. Although the effects of bicarbonate and CA activity on enhancing lactatedependent proton fluxes were modest in vitro (Table 1 ), the effects in vivo were quite striking.

Our hypothesis states that lactate: $\mathrm{H}^{+}$efflux is an osmotic component of the endothelial pump and that transendothelial flux is facilitated by intracellular and extracellular cellular buffering as illustrated in Figure 7. In vivo, we used four approaches to disrupt the components of the buffering system: intracameral injection of ouabain to inhibit the $\mathrm{Na}^{+}-\mathrm{K}^{+}$ ATPase, DIDS injection to inhibit anion transport including $\mathrm{Na}^{+}: 2 \mathrm{HCO}_{3}{ }^{-}$cotransport, NBCe1 shRNA to directly reduce $\mathrm{Na}^{+}: 2 \mathrm{HCO}_{3}{ }^{-}$expression and cotransport activity, and topical

TABLE 1. Lactate-Dependent $\mathrm{H}^{+}$Flux in Rabbit Corneal Endothelium

\begin{tabular}{lccccc}
\hline & $\boldsymbol{\beta}_{\mathbf{i}}$ & $\boldsymbol{\beta}_{\mathbf{H C O}}$ & $\boldsymbol{\beta}_{\mathbf{T}}$ & $\boldsymbol{\delta} \mathbf{p H i} / \mathbf{d t}$ & $\mathbf{j}_{\mathbf{H}+}(\mathbf{m M} / \mathbf{s e c})$ \\
\hline $\mathrm{BF}$ & 20 & 0 & 20 & 0.0016 & 0.032 \\
$\mathrm{BR}$ & 20 & 41 & 61 & 0.0006 & 0.0366 \\
$\mathrm{BF}+$ ACTZ & 20 & 0 & 20 & 0.0016 & 0.032 \\
BR+ACTZ & 20 & 23 & 43 & 0.0008 & 0.0344 \\
\hline
\end{tabular}




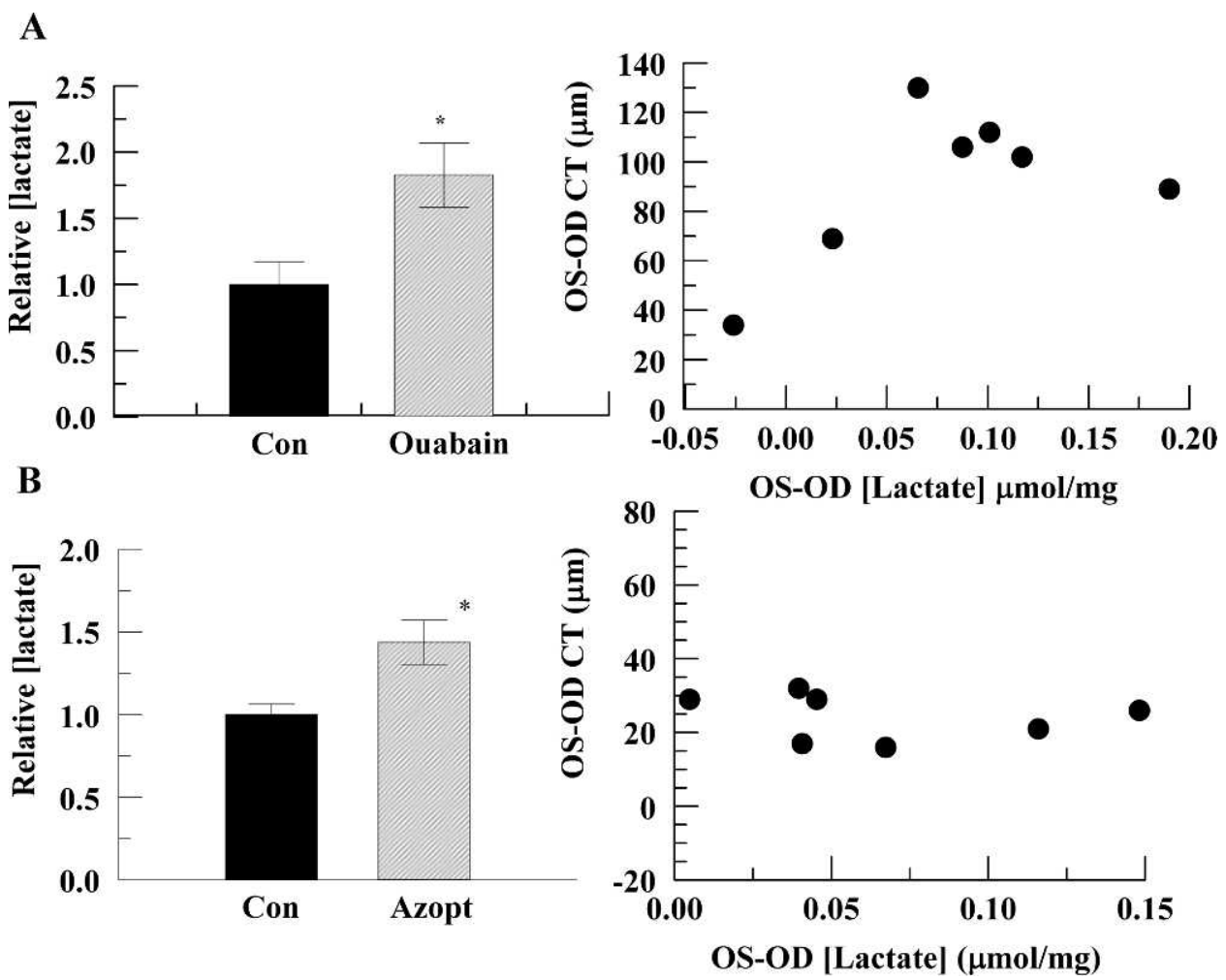

Figure 4. The effect of ouabain and topical CA inhibitor, Azopt, on stromal lactate concentration and central corneal thickness. (A) Ouabain (25 $\mu \mathrm{L}, 1 \mathrm{mM}$ ) was injected intracamerally into one eye and the other eye with an equal volume of PBS. The left bar graph shows the relative increase in lactate concentration of the ouabain-treated eyes. * Statistical significance at $P<0.05$. The right graph shows the relation between corneal swelling and stromal [lactate]. The $\mathrm{x}$-axis represents the difference in [lactate] between paired eyes. The [lactate] was normalized to protein concentration The y-axis represents the difference in the change in CT between the paired eyes. There was a moderate $(r=0.57)$ correlation between the extent of corneal swelling and [lactate] in eyes treated with ouabain, although this was not statistically significant. (B) Two drops of Azopt (1\%) were instilled on the surface of one eye and the other with PBS. Azopt produced a 1.43-fold increase in corneal [lactate] relative to the control eyes $(n=$ 7). The correlation between corneal swelling and [lactate] in eyes treated with Azopt was weak $(r=0.22)$. ${ }^{*}$ Statistical significance at $P<0.05$.

Azopt to inhibit CA activity. We predicted that each approach would cause an increase in corneal thickness and stromal [lactate]. This was true in all cases, but the relation between swelling and [lactate] varied depending on the approach.

As expected, ouabain induced significant corneal swelling (23\%) and increase in stromal [lactate] (Fig. 4A). Figure $4 \mathrm{~A}$ shows that increasing corneal swelling was associated with increasing stromal [lactate], but the correlation was not statistically significant. Azopt, on the other hand, produced a much smaller, but still significant increase in CT (approximate- ly 6\%-8\%). Again, swollen corneas had significantly more lactate; however, a direct correlation between change in CT and [lactate] could not be established. Similarly, DIDS induced significant corneal swelling and an increase in [lactate], but moderate correlation. Last, NBCe1 shRNA knockdown significantly increased CT and [lactate]. In contrast, there was a significant correlation between the change in CT and the increased [lactate]. In sum, all four approaches demonstrated a significant increase in corneal thickness and lactate concentration relative to control eyes. In most, there was an apparent
A

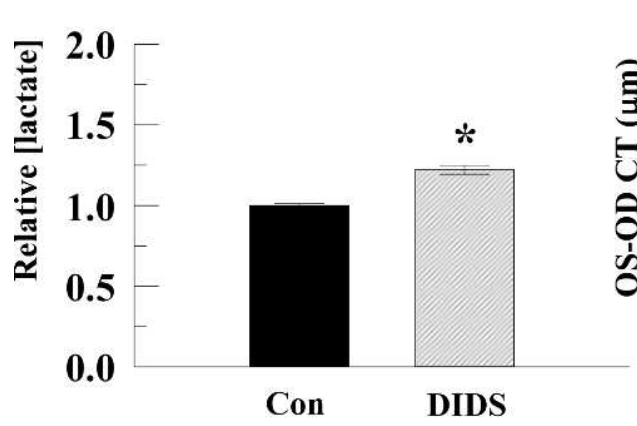

B

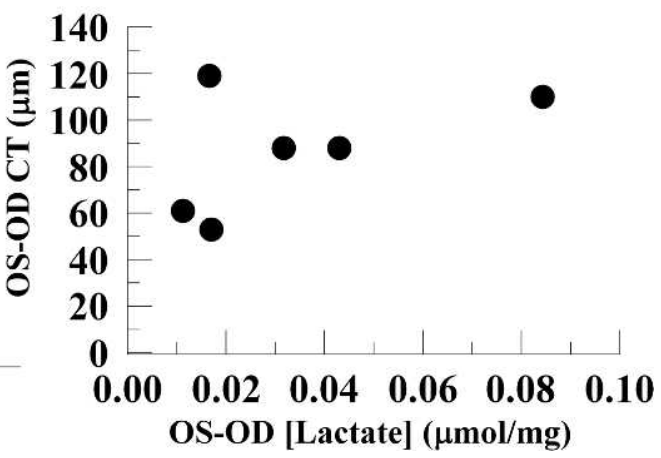

Figure 5. The effect of DIDS on stromal lactate concentration and central corneal thickness. DIDS (25 $\mu \mathrm{L}$, $10 \mathrm{mM})$ was injected intracamerally into one eye and the other with PBS. (A) DIDS induced a 1.25-fold greater corneal [lactate] compared with the untreated eye $(n=6)$. $P<0.05$. (B) There was a moderate $(r=0.50)$ correlation between corneal swelling and [lactate] in eyes treated with DIDS. * $P>0.05$. 
A

B
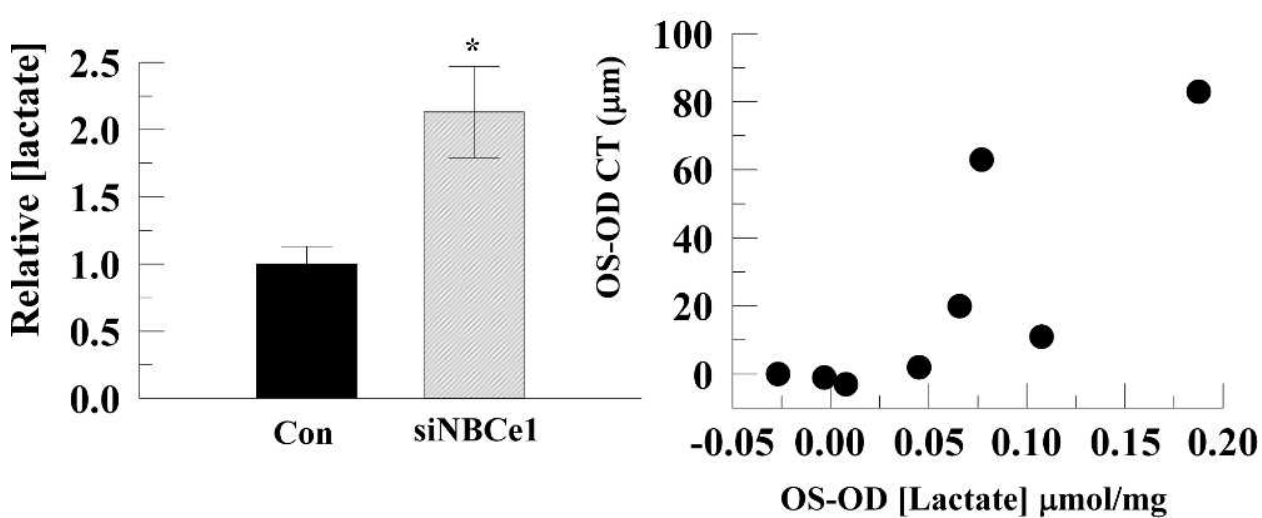

FiguRE 6. The effect of NBCe1 knockdown on stromal lactate concentration and central corneal thickness. NBCe1 shRNA was delivered intracamerally into one eye and the other eye received scrambled shRNA. (A) NBCe1 shRNA delivered with lentiviruses into the anterior chamber induced a greater than two-fold increase in corneal [lactate], $n=8, P<0.05$. (B) There was a strong correlation $(r=0.81)$ between corneal swelling and [lactate], $P<0.05$.

association between the degree of swelling and increased [lactate]. Whereas the acute experiments could not establish a significant correlation between swelling and [lactate], the chronic NBCe1 knockdown experiment did.

Corneal swelling can be produced by either inhibition of the pump or an increase in endothelial hydraulic conductivity (i.e., decreased barrier function). If the acute injections of inhibitors decrease barrier function (e.g., disruption of tight junctions), then swelling unrelated to the pump will occur. Possible disruption of barrier function could not be a result of the injections themselves, since the controls did not swell significantly. We do not expect ouabain or DIDS to disrupt barrier function; however, we used relatively high concentrations to offset dilution by aqueous humor. Variable mixing

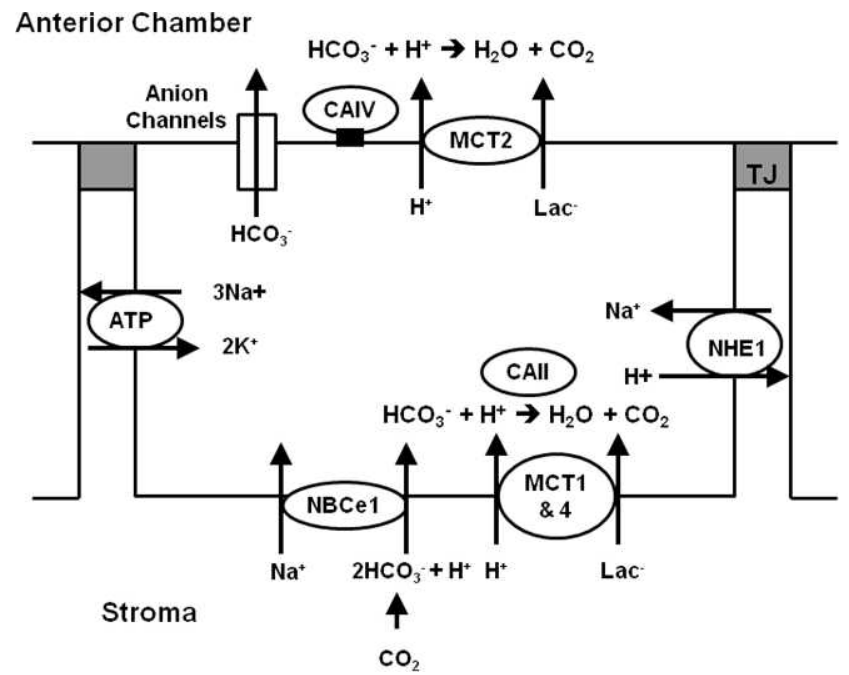

Figure 7. Facilitated lactate transport model. Lactate: $\mathrm{H}^{+}$is taken up at the basolateral (stromal) membrane by MCT1 and 4. The transported proton is converted to $\mathrm{CO}_{2}$ by combining with bicarbonate in a reaction catalyzed by carbonic anhydrase II. High intracellular $\left[\mathrm{HCO}_{3}{ }^{-}\right]$ is provided by robust basolateral NBCe1 activity. Buffering of the lactate-dependent $\mathrm{H}^{+}$influx or direct removal by $\mathrm{Na}^{+} / \mathrm{H}^{+}$exchange prevents $\mathrm{H}^{+}$accumulation and slowing of further lactate: $\mathrm{H}^{+}$influx. Efflux of lactate: $\mathrm{H}^{+}$at the apical membrane is provided by MCT2 Apical CA IV catalyzes buffering of ejected protons within the unstirred apical layer of the anterior chamber where $\mathrm{HCO}_{3}{ }^{-}$is readily available. following injection could expose the endothelium to the high concentrations that may have an effect on monolayer integrity. Similarly, topical Azopt is not expected to disrupt barrier function. However, the small amount of swelling with Azopt suggests that much larger numbers of experiments will be needed to show a correlation. If these approaches are used in the future, barrier function will need to be determined. ${ }^{46-48}$ We suspect that the knockdown approach is more robust in showing this relationship because any possible effects of injection solutions on barrier integrity are likely resolve over the weeks.

In summary, our results show that bicarbonate, bicarbonate transporters, and CA play a role in facilitating the clearance of lactate from the corneal stroma into the aqueous humor. Failure to efficiently remove lactate from the cornea will lead to corneal edema. Thus, the transport of lactate- $\mathrm{H}^{+}$is a significant component of the endothelial fluid pump. It is possible that lactate rather than $\mathrm{HCO}_{3}{ }^{-}$is the key ion that drives the endothelial fluid pump. This would be consistent with previous studies ${ }^{6,7,49-51}$ that show the endothelial fluid pump can still function in the absence of bicarbonate while in the presence of high buffer concentration. Further studies are needed to explore this link between lactate flux and fluid secretion in the corneal endothelium.

\section{Acknowledgments}

The authors thank Cailing Liu and Qiang Cheng for their technical assistance in designing the NBCe1 shRNA vector and producing the lentiviruses used in this study.

\section{References}

1. Riley MV, Winkler BS, Peters MI, Czajkowski CA. Relationship between fluid transport and in situ inhibition of $\mathrm{Na}(+)-\mathrm{K}+$ adenosine triphosphatase in corneal endothelium. Invest Ophthalmol Vis Sci. 1994;35:560-567.

2. Anderson EI, Fischbarg J. Biphasic effects of insulin and ouabain on fluid transport across rabbit corneal endothelium. J Physiol. 1978;275:377-389.

3. Wigham CG, Guggenheim JA, Hodson SA. Sodium movement into and out of corneal endothelium. Pflugers Archiv. 1994; 428:577-582. 
4. Hodson S, Miller F. The bicarbonate ion pump in the endothelium which regulates the hydration of rabbit cornea. J Physiol. 1976;263:563-577.

5. Fischbarg J, Lim J. Role of cations, anions, and carbonic anhydrase in fluid transport across rabbit corneal endothelium. J Physiol. 1974;241:647-675.

6. Riley M, Winkler B, Czajkowski C, Peters M. The roles of bicarbonate and $\mathrm{CO} 2$ in transendothelial fluid movement and control of corneal thickness. Invest Ophthalmol Vis Sci. 1995; 36:103-112.

7. Doughty MJ, Maurice D. Bicarbonate sensitivity of rabbit corneal endothelium fluid pump in vitro. Invest Ophthalmol Vis Sci. 1988;29:216-223.

8. Narula P, Xu M, Kuang K, Akiyama R, Fischbarg J. Fluid transport across cultured bovine corneal endothelial cell monolayers. Am J Physiol. 1992;262:C98-C103.

9. Kuang K, Xu M, Koniarek J, Fischbarg J. Effects of ambient bicarbonate, phosphate and carbonic anhydrase inhibitors on fluid transport across rabbit endothelium. Exp Eye Res. 1990; 50:487-493.

10. Guggenheim JA, Hodson SA. Localization of Na+/K(+)-ATPase in the bovine corneal endothelium. Biochim Biophys Acta. 1994;1189:127-134.

11. Jentsch TJ, Keller SK, Koch M, Wiederholt M. Evidence for coupled transport of bicarbonate and sodium in cultured bovine corneal endothelial cells. J Membr Biol. 1984;81:189204

12. Bonanno JA, Giasson C. Intracellular $\mathrm{pH}$ regulation in fresh and cultured bovine corneal endothelium. I. $\mathrm{Na}+/ \mathrm{H}+$ exchange in the absence and presence of HCO3. Invest Ophthalmol Vis Sci. 1992;33:3058-3067.

13. Sun XC, Bonanno JA, Jelamskii S, Xie Q. Expression and localization of $\mathrm{Na}(+)-\mathrm{HCO}(3)(-)$ cotransporter in bovine corneal endothelium. Am J Physiol Cell Physiol. 2000;279: C1648-C1655.

14. Bonanno JA, Guan Y, Jelamskii S, Kang XJ. Apical and basolateral $\mathrm{CO} 2-\mathrm{HCO} 3-$ permeability in cultured bovine corneal endothelial cells. Am J Physiol. 1999;277(pt 1): C545-C553.

15. Jelamskii S, Sun XC, Herse P, Bonanno JA. Basolateral $\mathrm{Na}(+)-$ $\mathrm{K}(+)-2 \mathrm{Cl}(-)$ cotransport in cultured and fresh bovine corneal endothelium. Invest Ophthalmol Vis Sci. 2000:488-495.

16. Jentsch TJ, Korbmacher C, Janicke I, et al. Regulation of cytoplasmic $\mathrm{pH}$ of cultured bovine corneal endothelial cells in the absence and presence of bicarbonate. J Membr Biol. 1988; 103:29-40.

17. Bonanno JA, Yi G, Kang XJ, Srinivas SP. Reevaluation of Cl-/ HCO3- exchange in cultured bovine corneal endothelial cells. Invest Ophthalmol Vis Sci. 1998;39:2713-2722.

18. Sun XC, McCutheon C, Bertram P, Xie Q, Bonanno JA. Studies on the expression of mRNA for anion transport related proteins in corneal endothelial cells. Curr Eye Res. 2001;22: $1-7$.

19. Hodson S, Miller $F$. The bicarbonate ion pump in the endothelium which regulates the hydration of rabbit cornea. J Physiol. 1976;263:563-577.

20. Maurice DM. Passive ion fluxes across the corneal endothelium. Curr Eye Res. 1985;4:339-349.

21. Bonanno JA, Srinivas SP. Cyclic AMP activates anion channels in cultured bovine corneal endothelial cells. Exp Eye Res. 1997;64:953-962.

22. Sun XC, Zhai CB, Cui M, et al. $\mathrm{HCO}(3)(-)$-dependent soluble adenylyl cyclase activates cystic fibrosis transmembrane conductance regulator in corneal endothelium. Am J Physiol Cell Physiol. 2003;284:C1114-C1122.

23. Bonanno JA. Molecular mechanisms underlying the corneal endothelial pump. Exp Eye Res. 2011;2:95(1):2-7.
24. Nguyen TT, Bonanno JA. Bicarbonate, NBCe1, NHE, and carbonic anhydrase activity enhance lactate- $\mathrm{H}+$ transport in bovine corneal endothelium. Invest Ophthalmol Vis Sci. 2011; 52:8086-8093.

25. Riley MV. Glucose and oxygen utilization by the rabbit cornea. Exp Eye Res. 1969;8:193-200.

26. Klyce SD. Stromal lactate accumulation can account for corneal oedema osmotically following epithelial hypoxia in the rabbit. J Physiol. 1981;321:49-64.

27. Liu C, Cheng Q, Nguyen T, Bonanno JA. Knockdown of NBCe1 in vivo compromises the corneal endothelial pump. Invest Ophthalmol Vis Sci. 2010;51:5190-5197.

28. Roos A, Boron WF. Intracellular pH. Physiol Rev. 1981;61:296434

29. Giasson C, Bonanno JA. Corneal epithelial and aqueous humor acidification during in vivo contact lens wear in rabbits. Invest Ophthalmol Vis Sci. 1994;35:851-861.

30. Bonanno JA, Polse KA. Measurement of in vivo human corneal stromal pH: open and closed eyes. Invest Ophthalmol Vis Sci. 1987;28:522-530.

31. Castorino JJ, Deborde S, Deora A, et al. Basolateral sorting signals regulating tissue-specific polarity of heteromeric monocarboxylate transporters in epithelia. Traffic. 2011;12: 483-498.

32. Kirat D, Kato S. Monocarboxylate transporter genes in the mammary gland of lactating cows. Histochem Cell Biol. 2009; 132:447-455.

33. Kirat D, Matsuda Y, Yamashiki N, Hayashi H, Kato S. Expression, cellular localization, and functional role of monocarboxylate transporter 4 (MCT4) in the gastrointestinal tract of ruminants. Gene. 2007;391:140-149.

34. Kirat D, Sallam K, Hayashi H, Miyasho T, Kato S. Presence of ten isoforms of monocarboxylate transporter (MCT) family in the bovine adrenal gland. Mol Cell Endocrinol. 2009;298:89100 .

35. Halestrap AP, Price NT. The proton-linked monocarboxylate transporter (MCT) family: structure, function and regulation. Biochem J. 1999;343 (pt 2):281-299.

36. Halestrap AP, Wilson MC. The monocarboxylate transporter family-role and regulation. IUBMB Life. 2011;2:64:(2)109119.

37. Jackson VN, Halestrap AP. The kinetics, substrate, and inhibitor specificity of the monocarboxylate (lactate) transporter of rat liver cells determined using the fluorescent intracellular $\mathrm{pH}$ indicator, 2', $2^{\prime}$-bis(carboxyethyl)-5(6)-carboxyfluorescein. J Biol Chem. 1996;271:861-868.

38. Hartzell C, Putzier I, Arreola J. Calcium-activated chloride channels. Annu Rev Physiol. 2005;67:719-758.

39. Becker HM, Deitmer JW. Nonenzymatic proton handling by carbonic anhydrase II during $\mathrm{H}+$-lactate cotransport via monocarboxylate transporter 1. J Biol Chem. 2008;283: 21655-21667.

40. Wetzel P, Hasse A, Papadopoulos S, Voipio J, Kaila K, Gros G. Extracellular carbonic anhydrase activity facilitates lactic acid transport in rat skeletal muscle fibres. $J$ Physiol. 2001;531(pt 3):743-756.

41. Svichar N, Chesler M. Surface carbonic anhydrase activity on astrocytes and neurons facilitates lactate transport. Glia. 2003; 41:415-419.

42. Becker HM, Broer S, Deitmer JW. Facilitated lactate transport by MCT1 when coexpressed with the sodium bicarbonate cotransporter (NBC) in Xenopus oocytes. Biophys J. 2004; 86(pt 1):235-247.

43. Becker HM, Hirnet D, Fecher-Trost C, Sultemeyer D, Deitmer JW. Transport activity of MCT1 expressed in Xenopus oocytes is increased by interaction with carbonic anhydrase. $J$ Biol Chem. 2005;280:39882-39889. 
44. Becker HM, Klier M, Deitmer JW. Nonenzymatic augmentation of lactate transport via monocarboxylate transporter isoform 4 by carbonic anhydrase II. J Membr Biol. 2010;234:125-135.

45. Becker HM, Deitmer JW. Voltage dependence of $\mathrm{H}+$ buffering mediated by sodium bicarbonate cotransport expressed in Xenopus oocytes. J Biol Chem. 2004;279:28057-28062.

46. Bourne WM, Hodge DO, McLaren JW. Estimation of corneal endothelial pump function in long-term contact lens wearers. Invest Opbthalmol Vis Sci. 1999;40:603-611.

47. Carlson KH, Bourne WM. The clinical measurement of endothelial permeability. Cornea. 1988;7:183-189.
48. Bourne WM. Clinical estimation of corneal endothelial pump function. Trans Am Opbthalmol Soc. 1998;96:229-239; discussion 39-42.

49. Doughty MJ. Evidence for a direct effect of bicarbonate on the rabbit corneal stroma. Optom Vis Sci. 1991;68:687-698.

50. Doughty MJ. New observations on bicarbonate-pH effects on thickness changes of rabbit corneas under silicone oil in vitro. Am J Optom Physiol Opt. 1985;62:879-888.

51. Doughty MJ, Newlander K, Olejnik O. Effect of bicarbonatefree balanced salt solutions on fluid pump and endothelial morphology of rabbit corneas in-vitro. J Pharm Pharmacol. 1993;45:102-109. 